\title{
Science camera calibration for extreme adaptive optics
}

James Kent Wallace, Joseph J. Green, Michael Shao, Mitchell Troy, James P. Lloyd, et al.

James Kent Wallace, Joseph J. Green, Michael Shao, Mitchell Troy, James P. Lloyd, Bruce Macintosh, "Science camera calibration for extreme adaptive optics," Proc. SPIE 5490, Advancements in Adaptive Optics, (25 October 2004); doi: $10.1117 / 12.552362$

SPIE Event: SPIE Astronomical Telescopes + Instrumentation, 2004, Glasgow, United Kingdom 


\title{
Science Camera Calibration for Extreme Adaptive Optics
}

\author{
J. Kent Wallace ${ }^{* a}$, Joseph J. Green ${ }^{\mathrm{a}}$, Michael Shao ${ }^{\mathrm{a}}$, Mitchell Troy, \\ James Lloyd ${ }^{\mathrm{b}}$, Bruce MacIntosh ${ }^{\mathrm{c}}$ \\ ${ }^{a}$ Jet Propulsion Laboratory, California Institute of Technology, 4800 Oak Grove Dr., \\ Pasadena, CA 91109 \\ ${ }^{\mathrm{b}}$ California Institute of Technology, 1200 East California Blvd., Pasadena, CA 91125 \\ ${ }^{c}$ Lawrence Livermore National Laboratory, 7000 East Ave., Livermore, CA, 94550
}

\begin{abstract}
The nascent field of planet detection has yielded a host of extra-solar planet detections. To date, these detections have been the result of indirect techniques: the planet is inferred by precisely measuring its effect on the host star. Direct observation of extra-solar planets remains a challenging yet compelling goal. In this vein, the Center for Adaptive Optics has proposed a ground-based, high-actuator density extreme AO system (XAOPI), for a large $(\sim 10 \mathrm{~m})$ telescope whose ultimate goal is to directly evidence a specific class of these objects: young and massive planets. Detailed system wave-front error budgets suggest that this system is a feasible, if not an ambitious, proposition. One key element in this error budget is the calibration and maintenance of the science camera wave front with respect to the wave-front sensor which currently has an allowable contribution of $\sim 5$ nanometers rms.

This talk first summarizes the current status of calibration on existing ground-based AO systems, the magnitude of this effect in the system error budget and current techniques for mitigation. Subsequently, we will explore the nature of this calibration error term, it's source in the non-commonality between the science camera and wave front sensor, and the effect of the temporal evolution of non-commonality. Finally, we will describe preliminary plans for sensing and controlling this error term. The sensing techniques include phase retrieval, phase contrast and external metrology. To conclude, a calibration scenario that meets the stringent requirement for XAOPI will be discussed.
\end{abstract}

Keywords: extreme adaptive optics, calibration, extra-solar planet detection

\section{INTRODUCTION}

\section{The High Contrast Calibration Architecture}

Canonical AO systems ${ }^{1}$ introduce significant amounts of non-commonality between the location where the wave front is sensed for adaptive correction (the WFS) and the location of the science observation (the SC). This non-commonality is broad and deep: it is spatial, temporal and chromatic.

The WFS and SC are located on an optical bench and are typically separated by a half-meter, or so, and have roughly a meter of longitudinal non-common optical path length. This spatial separation leads to differential effects that are measured by the WFS and incorrectly applied to the DM and hence the SC. Flexure of the optical table, for instance, will result in bore sight differences between the WFS and SC over time.

Chromatic errors are introduced due to the difference in science and wave front sensing wavelengths. Under these circumstances, the beam train as seen by the WFS wavelength is different than the beam train as seen by the $\mathrm{SC}$ wavelength. For instance, as the zenith angle grows, atmospheric dispersion will cause

* James.K.Wallace@jpl.nasa.gov, Phone:1.818.393.7066, fax: 1.838.393.4357 
differential chromatic beam walk on the optics not located at a pupil. A pair of Risley prisms can be introduced into the system to compensate for some of these errors, but cannot compensate for elements that occur previous to the prism pairs.

Temporal errors are introduced by the different sampling periods of the WFS and SC. The WFS typically samples at a few hundred hertz, while the SC is an integrating camera. Therefore, the introduction of noncommon path errors into the science camera is not sensed frequently enough in the right location and in the right wavelength to be corrected.

A high contrast observation for extra-solar planet detection ${ }^{2}$ requires a fundamental shift in thinking with regards to the instrument performance, calibration and, in turn, the optical configuration. In order to combat the effects of this non-commonality, we will employ a fundamental shift in $\mathrm{AO}$ architecture. We propose a tight integration between: 1) the coronagraph, 2) a device we call the precision wave front sensor (PWFS) and 3) the science camera. The calibration camera will share the same optical band, and the collocation of all these instruments minimizes the spatial non-commonality.

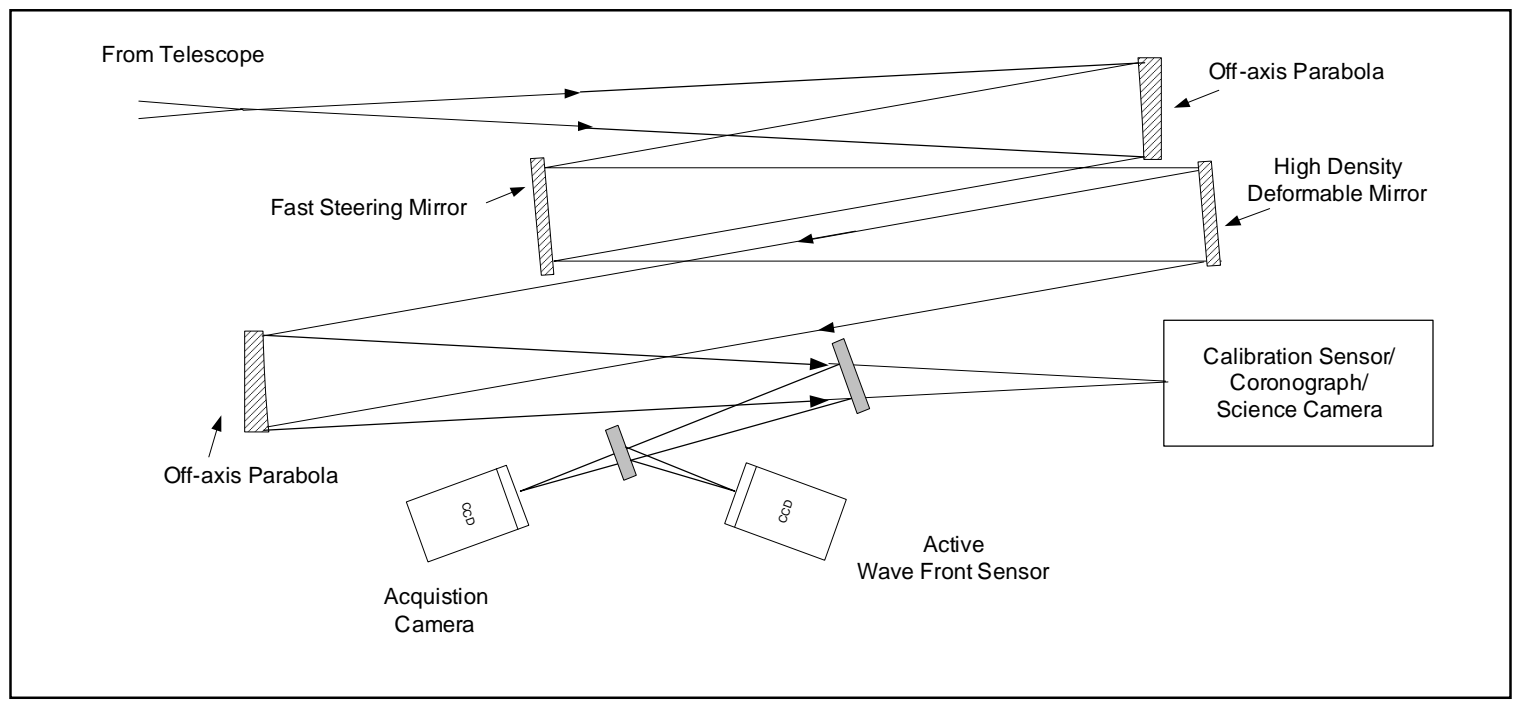

Fig. 1. Illustration of a high-contrast adaptive optics system with integrated coronagraph, calibration sensor, and science camera.

\section{Calibration Scenarios}

Try as we might to mitigate all non-commonality, ultimately there will be some residual non-common path errors. Therefore, one is left with the secondary goal of using a precision WFS (PWFS) that is a proxy to the wave front seen by the science camera. The goal of the calibration routine is twofold: 1) to make a relative measurement of the wave front between the PWFS and the wave front seen by the science camera and 2) to maintain this wave front during a period of observation.

The relative calibration routine occurs during non-observational periods (daytime for instance) when the instrument will be feed with a stimulus that simulates the pupil and image planes of the telescope with a couple of different, selectable, sources (thermal and laser). The wave front is measured at the science camera in a fashion that optimizes the coronagraph performance. Here we plan to use techniques developed on the HCIT such as focus diversity and speckle nulling. The wave front optimization is done via the DM which is updated by giving new centroid offsets to the active wave front sensor. Once this is satisfactory, the wave front is measured with the high-contrast wave front sensor. It's this wave front sensor set point that will be used during the science observation. 
The maintenance calibration routine during a science observation consists of simply measuring the wave front on the PWFS and providing centroid offsets to the active WFS. In this manor, the PWFS is proxy to the science camera wave front.

\subsection{Calibration Architectures}

The basis of the calibration method is to compare the wave front to be measured (the unknown) with one that serves as a reference. The difference between the two serves as a signal that can be used to drive the system to a desirable set point. (The reference doesn't necessarily need to be a perfect wave front, it needs to remain stable at the level to which control is required.) All of the proposed techniques investigated have this property of a reference wave front that is generated in order to compare it with the unknown wave front. Before the coronagraph, the reference is generated from the low spatial frequency component of some fraction of light taken from the science beam. We'll describe how this is done in a short while. In this mode, we have the benefit of photons, but pay the price in non-commonality. This architecture is shown, schematically, in Fig. 2.

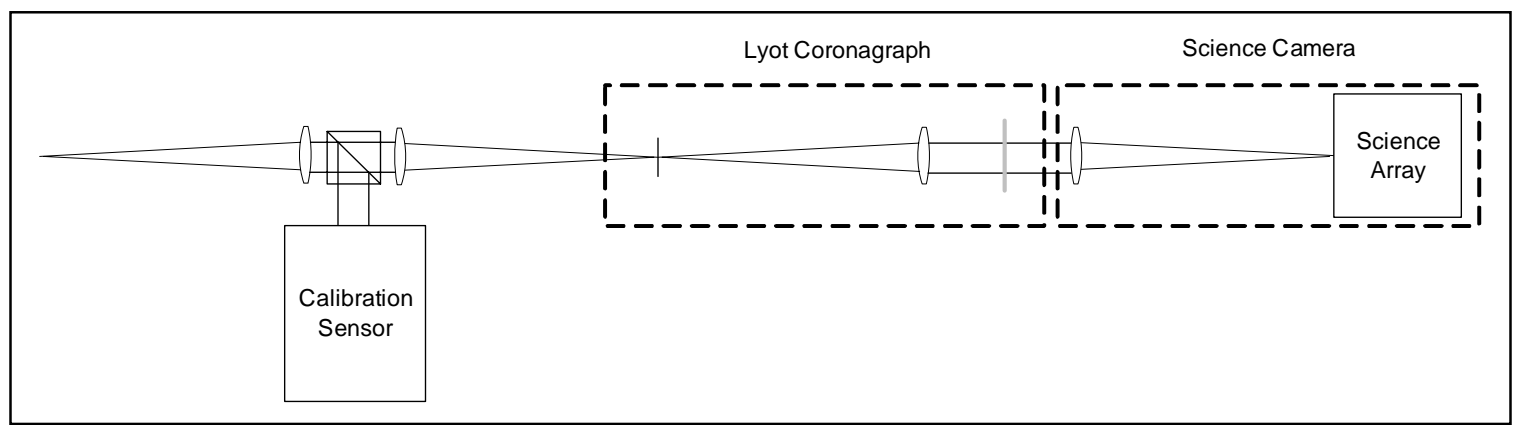

Fig. 2. Calibration sensor before the coronagraph and science camera.

After the Lyot Coronagraph, the low spatial frequency is no longer present, and therefore cannot be used to create the reference wave front. In this scenario, the occulting spot itself acts as a reference and can be used to measure the residual errors. This will be described in more detail subsequently. The architecture is shown schematically in Fig. 3.

Several different types of calibration schemes, both before and after the coronagraph, have been investigated. Using a list of discriminators, we have arrived at a method we believe is most promising. As part of this decision making process, we'll describe the discriminators and justify our selection.

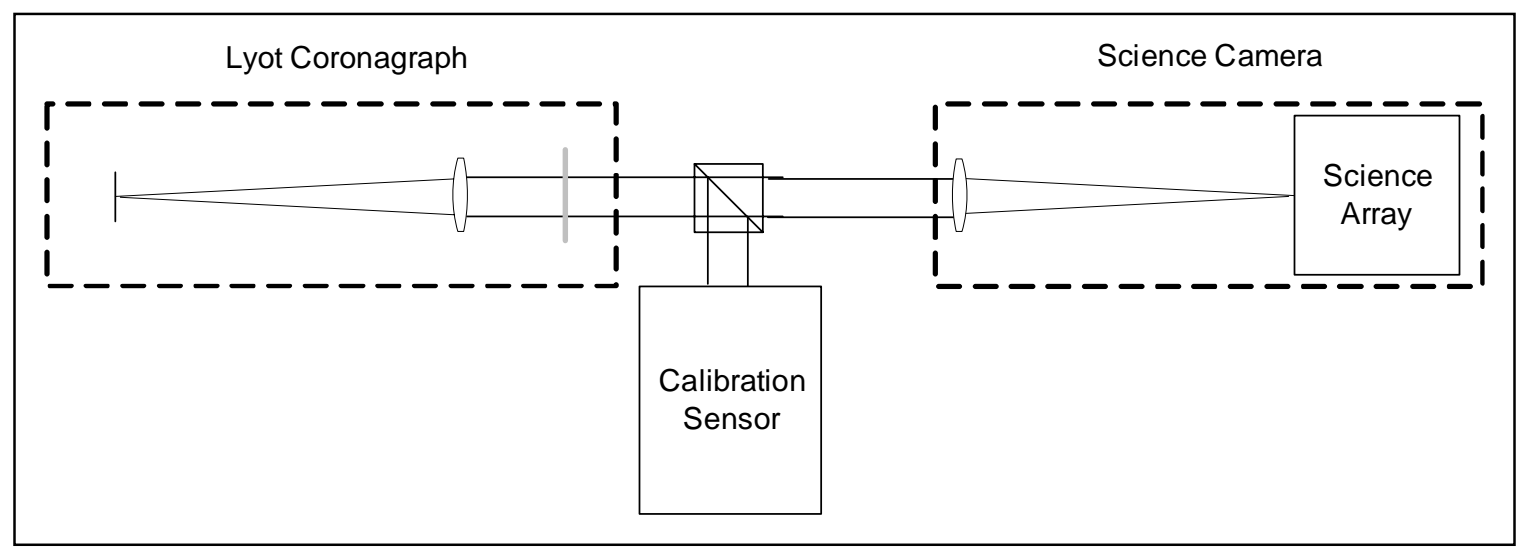

Fig. 3. Calibration sensor located after the Lyot coronagraph and before the science camera. 


\section{PRE-CORONAGRAPH WAVE FRONT SENSING}

\subsection{Phase Shifting Interferometer}

In this proposed method, light from before the Lyot coronagraphs is split and diverted to the precision wave front sensor. This light is then split again to form two beams of a Mach-Zehnder Interferometer. Both arms pass through a pair of lenses; one arms passes through a pinhole that serves as a spatial filter. At recombination, the reference wave front is combined with the unknown $\mathrm{arm}^{3}$. By employing the standard methods of phase shifting interferometry ${ }^{4}$, its possible to measure the wave front phase and amplitude errors.

This method has several advantages. It's before the Lyot coronagraph, so there are plenty of photons to use in order to create the reference wave front of the interferometer. (These photons would otherwise be lost behind the occulting spot). This technique allows one to not only solve for the magnitude and sign of the phase errors, it also permits the solution of the amplitude variance across the pupil as well.

A drawback is that this system, as currently envisioned in Fig. 4, requires a modulation of the path length in one arm. This modulation, if not done properly, can introduce errors in the phase measuring process. A means of phase shifting that is not susceptible to the telescope vibrations would need to be considered. These methods do exist, but can quickly complicate the PWFS with additional components.

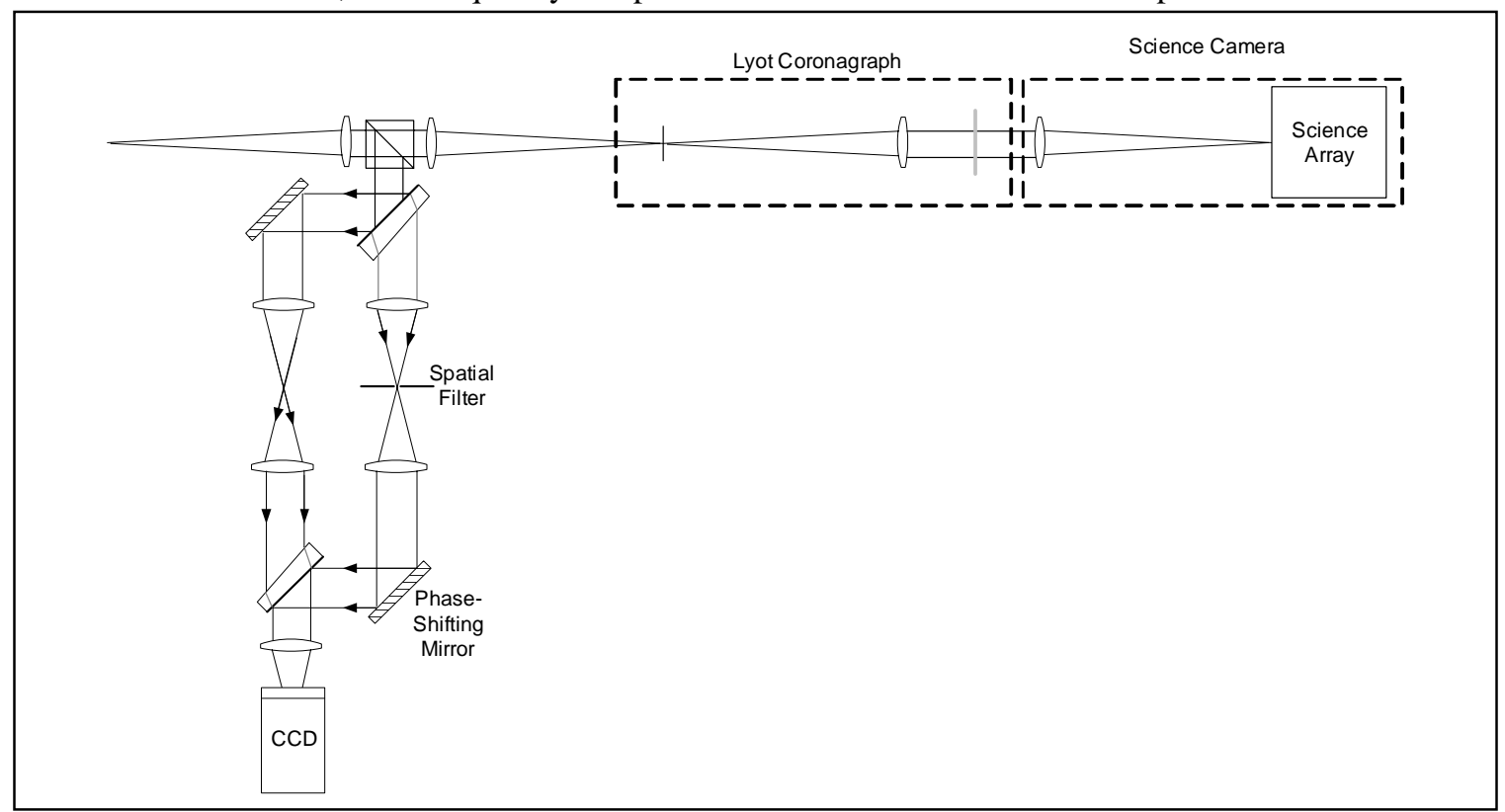

Fig. 4. Phase-shifting interferometer sensor for calibration.

The phase-shifting PWFS still suffers from residual non-common path that will produce un-sensed errors. In particular, the pinhole spatial filter that creates the reference wave front of the interferometer must be coaligned and maintained during a science observation. This would require yet another set in the calibration process.

\subsection{Phase contrast technique}

The residual wave front errors, post $\mathrm{AO}$ correction can be written as:

$$
t=e^{i \phi(x, y)}
$$


If we assume the residual phase errors are small, then a Taylor series expansion of the wave front illustrates that the first order and second order elements are out of phase by $\pi / 2$ radians.

$$
t \cong 1+i \varphi(x, y)
$$

Introducing a $\pi / 2$ phase shift for the first-order component of the beam will bring these two terms back in phase with one another, and allow them to interfere. This is done by forming a focus, and centering the resulting point spread function on a dielectric spot that introduces the additional $\pi / 2$ phase change. This spot is roughly the size of the $\lambda . \mathrm{f} / \#$. This can be expressed as:

$$
\mathfrak{I}[t]=\delta(f x) \delta(f y) e^{i \pi / 2}+\mathfrak{I}[i \varphi(x, y)]=i \delta(f x) \delta(f y)+\mathfrak{I}[i \varphi(x, y)]
$$

At a re-imaged pupil plane, the resulting wave front can be expressed as:

$$
E \cong \mathfrak{I}^{-1}[i \delta(f x) \delta(f y)+\mathfrak{I}[i \varphi(x, y)]] \cong i\left[1+\varphi\left(x^{\prime}, y^{\prime}\right)\right]
$$

The intensity distribution is then:

$$
I \cong\left|i\left[1+\varphi\left(x^{\prime}, y^{\prime}\right)\right]\right|^{2} \cong 1+2 \varphi\left(x^{\prime}, y^{\prime}\right)
$$

The original phase errors are linearly translated into intensity errors that reside on a constant background ${ }^{5}$. In this manor, a straightforward measurement of the intensity fluctuations gives a direct measure of the phase fluctuations across the pupil.

Since this concept has no moving parts, it is largely insensitive to the vibration environment of the telescope. Likewise, since the measurement is only intensity, the reconstruction to get to the phase is a single multiply that can be done quite quickly. Since the phase measurement is directly mapped to the the DM actuators, the reconstruction can be easily pipelined (only the pixels corresponding to a DM element need to be read out before the reconstruction can begin.) The idea of introducing a phase-shift spot in the focal plane was first proposed by Zernike ${ }^{6}$.

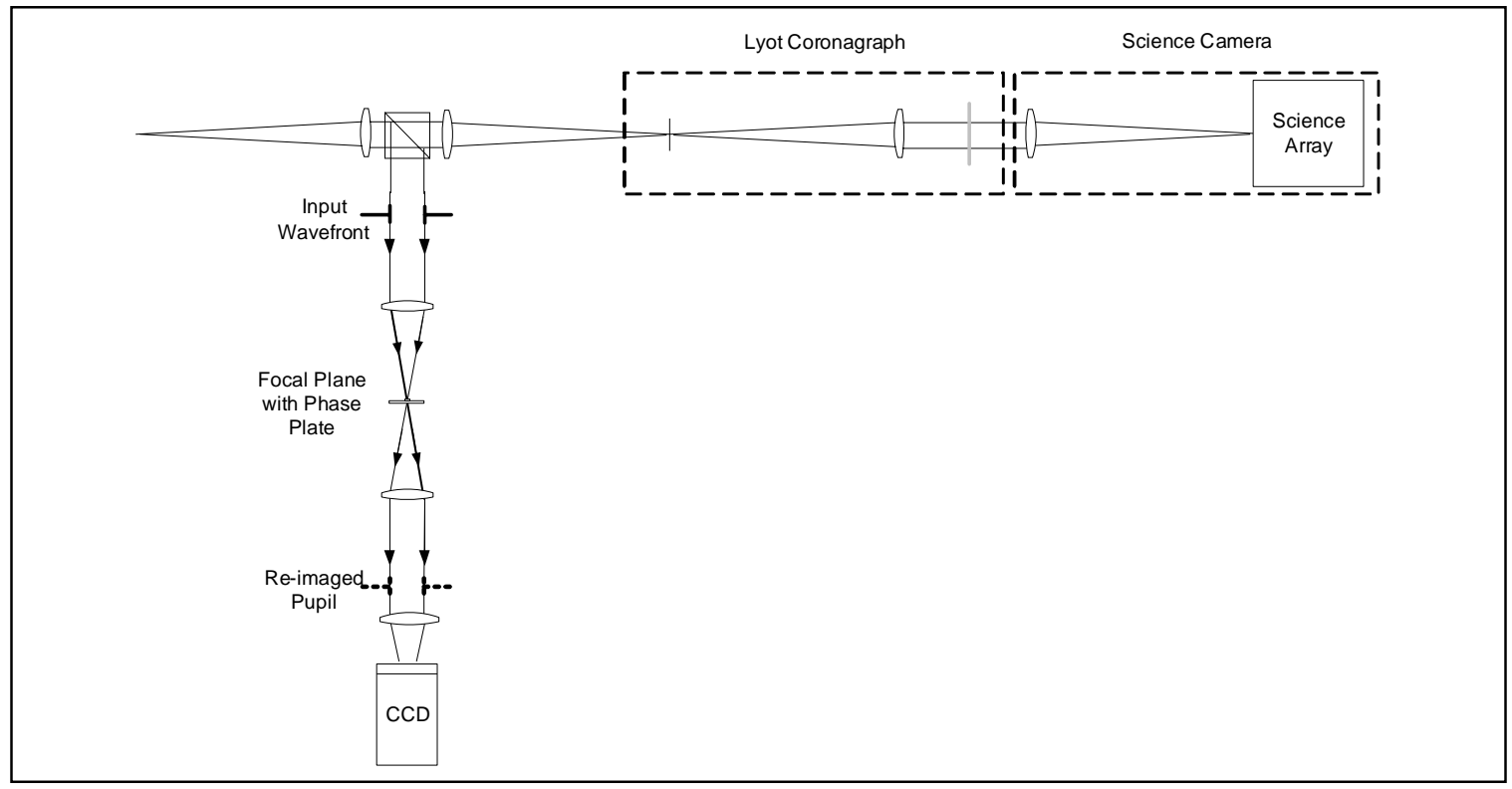

Fig. 5. Phase-contrast calibration sensor located before the coronagraph and science camera. 
Part of the simplicity is also part of the drawback of this method. Since it is assumed that a variation in intensity in the re-imaged pupil is due to phase effects, any other source of intensity fluctuation (atmospheric scintillation and variations in coating reflectivity) will misinterpreted as phase.

Like the phase-shifting interferometer approach, the system suffers from residual non-common path errors. In particular, the diffraction limited phase spot much be well aligned to the occulting spot in the Lyot coronagraph.

\section{POST CORONAGRAPH WAVE FRONT SENSING}

A quick discussion of the coronagraph's effect on residual wave front errors is in order. In a post-adaptive optics correction scenario, the wave front can be expanded as shown previous in Eq. 2. Starting with such an expression, the intensity distribution that is created in bringing the light to a focus is given by Eq. 6 .

$$
\mathfrak{I}[t]=\delta(f x) \delta(f y)+\mathfrak{I}[i \varphi(x, y)]
$$

The effect of the occulting spot is to remove the first order delta function (the core of the PSF). The electric field at the re-imaged pupil is given by:

$$
E \cong \mathfrak{I}^{-1}[\mathfrak{I}[i \varphi]] \cong i \varphi\left(x^{\prime}, y^{\prime}\right)
$$

And the corresponding intensity distribution is:

$$
I=\left|i \varphi\left(x^{\prime}, y^{\prime}\right)\right|^{2} \cong \varphi\left(x^{\prime}, y^{\prime}\right)^{2}
$$

Post-coronagraph, at the re-imaged pupil, the resultant intensity fluctuations are proportional to the square of the initial, residual phase errors.

If one were to take a fraction of the science light post-coronagraph, and re-image the pupil, its possible to have a very sensitive means of measuring the residual wave front errors. A schematic for such a system is shown in Fig. 6. Real data illustrating this effect is shown in Fig. 7. This data is taken from the High Contrast Imaging Testbed Facility at JPL.

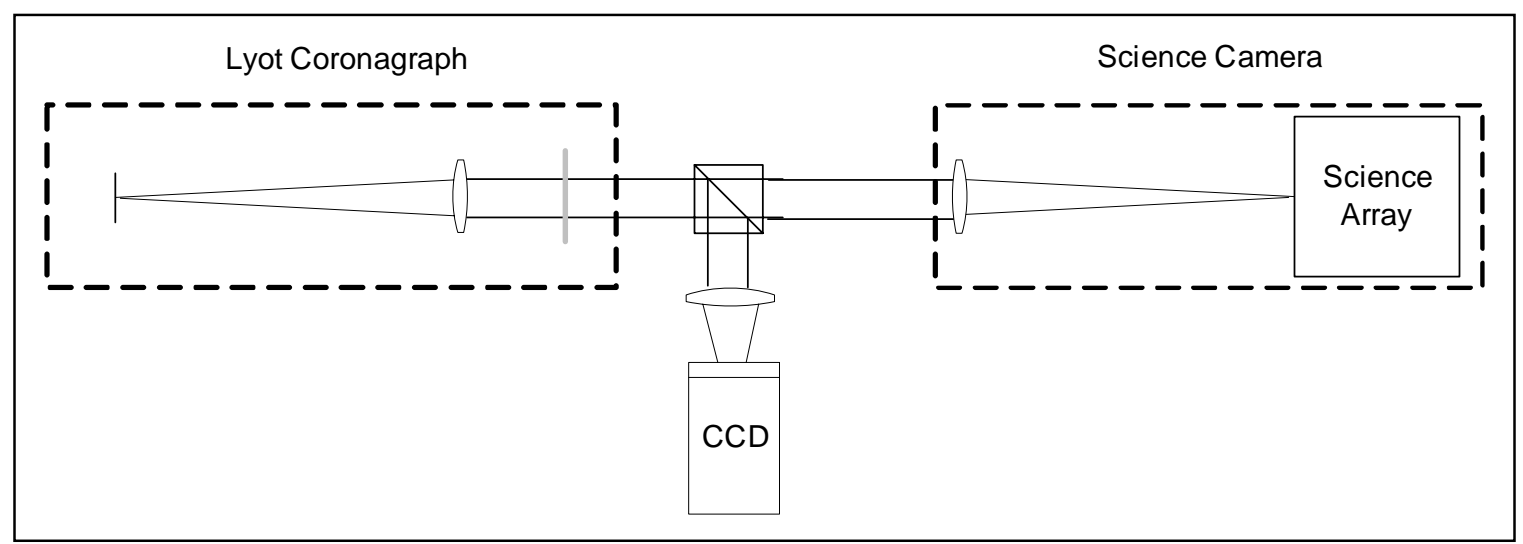

Fig. 6. Post-coronagraph wave front sensing. 


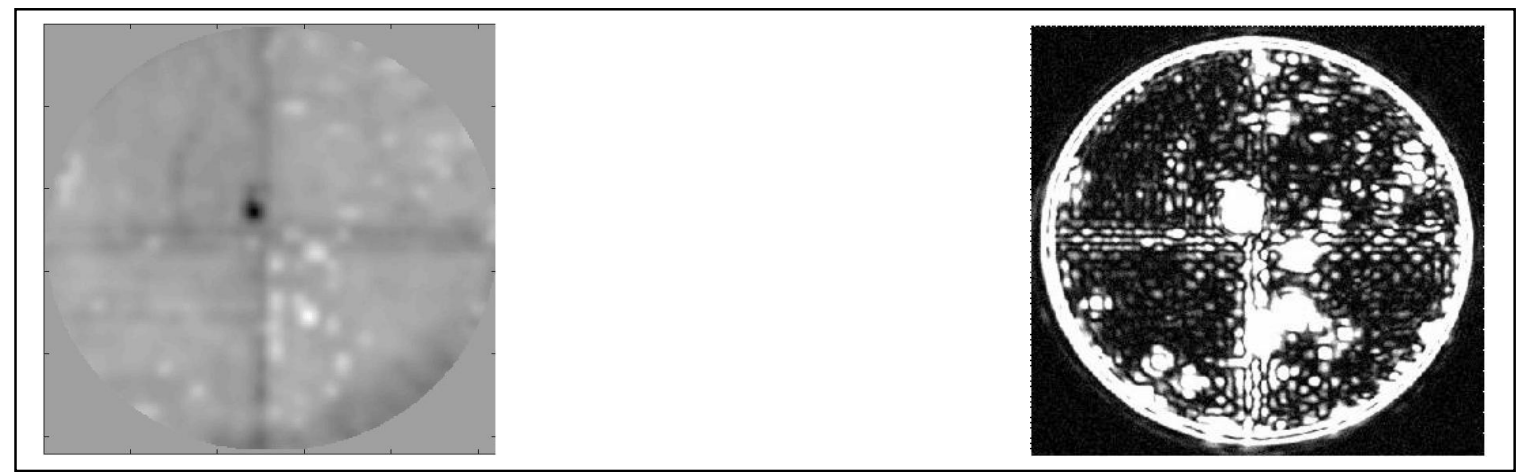

Fig. 7 The effect of residual phase errors (post-coronagraph) on the intensity distribution in the re-imaged pupil.

This technique is ideal in that it measures the residual errors post-coronagraph. Nulling out these pupil speckles is identical to minimizing the residual wave front errors. The difficulty with this method is that information regarding the sign of the phase has been lost. A means of introducing diversity is required that would somehow allows us to modulate the phase while simultaneously observing the effect on the corresponding bright speckle in the re-imaged pupil. This diversity must be introduced either before the coronagraph or internal to it. Wee have explored both of these techniques.

\subsection{Diversity before the coronagraph}

The easiest place to modulate the pupil phase is at the deformable mirror. By dithering an actuator at this plane, and determining if the corresponding pupil speckle gets brighter or dimmer, its possible to solve for the optimum location of the actuator to darken the speckle and thereby improve the residual wave front error.

The principles of this method have been demonstrated on both the Palomar Adaptive Optics System and the HCIT. The Palomar system demonstrated the principle on a single DM actuator, and the tests on the HCIT demonstrated a method of solving for a host of DM actuators simultaneously ${ }^{7}$.

This approach is seen as somewhat onerous due to the disruptive control on the DM. During a science observation, this dither would be in addition to the update commands sent from the active wave front sensor.

\subsection{Diversity internal to the Lyot coronagraph}

Recently, a new technique has been devised that introduces diversity internal to the Lyot coronagraph. This diversity, similar to the deformable mirror actuator diversity discussed previously, provides the extra information that is needed to solve for the residual phase errors unambiguously. In this method, the occulting spot of the Lyot Coronagraph is dithered and the resulting modulation in the pupil speckles is recorded. The dithering must be done at a level that leaves the planet contrast unaffected, yet still allows one the sensitivity to remove the residual wave front errors.

This approach allows us to measure the wave front post coronagraph, so we have mitigated the effects of non-commonality. Unfortunately, this method suffers from the need to dither the occulting spot.

\subsection{Hybrid Techniques}

A method that combines the best of pre and post coronagraph methods is shown in Fig. 8. In this technique, the low-spatial frequency photons that would otherwise be removed by the occulting spot are reflected via a diffraction limited spot. The resulting wave front, since it has been spatially filtered, is used as the reference wave front. A fraction of the science light, post occulter (but before the Lyot pupil mask) is diverted and combined with the reference wave front as mentioned previously. In this method, we benefit 
from the use of the low spatial frequency light that would otherwise be lost, and we employ phase shifting interferometry that allows us to measure both amplitude and phase errors.

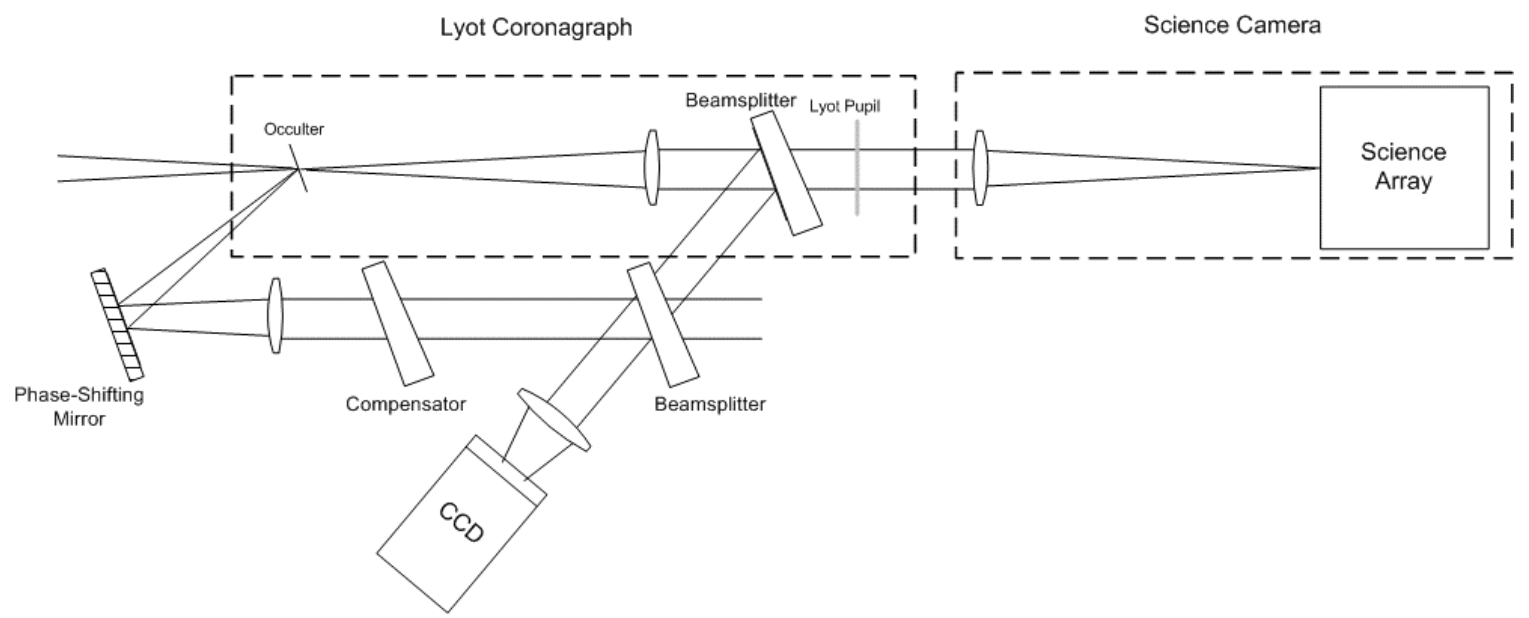

Fig. 8 Hybrid technique for measuring post-coronagraph errors using pre-coronagraph photons for the reference generation.

The resulting amplitude and phase measurement can be used in a couple of ways. First, it can be used as a "knowledge" sensor that allows us to know the speckle noise in the science camera image plane (calculated by taking an FFT of the amplitude and phase measurement). This information can be used to later subtract out these errors form the science observation.

Alternatively, it may be possible to use the phase measurement to assert an additional level of control to the high-density deformable mirror. The phase measurements can be done very quickly and applied to the DM on the order of a few milliseconds.

This method has several of the advantages of both the pre and post coronagraph sensing techniques. It measures the wave front errors post coronagraph so that non-common path errors are mitigated. It uses a reference wave front that is created from the photons that occur before the occulting spot and would otherwise be attenuated. And, it permits a rapid measurement of both phase and amplitude effects. All of this is done in the same optical band as the science camera, so chromatic errors are also mitigated. The method also allows us to measure the residual errors contemporaneous to a science observation, so that no interruption for calibration is required.

\section{PRECISION WAVE FRONT SENSOR SELECTION}

A host of proposed precision wave front sensors has been presented. A selection process is in order to permit us to choose the most desirable option. We will employee a decision matrix to guide our thinking and efficiently convey the basis of our ultimate decision. This method is commonly used at JPL in similar decision-making processes. Lacking room for a detailed explanation, it can be quickly summarized as numerical means of arriving at the best solution based upon a weighting of the desired properties (assuming each method under consideration meets the requirements.)

From our decision matrix its evident that post-coronagraph methods are most attractive. They measure errors where their impact is evident and at a location where the non-common path errors are zero. By using a fraction of the science photons, chromatic errors introduced from sensing at a different wavelength are also zeroed out. And finally, this precision wave front sensing is done contemporaneously with the science observation. In this way, we can be guaranteed that errors that would otherwise occur during periods of intermittent calibrations are also nulled. 
Further study of these techniques, both analytically and numerically, is in order to determine the sensor most suitable for the high-contrast imaging from the ground. We will judge the proposed techniques against the wave front calibration and maintenance requirements, Time permitting; we shall test these techniques to increase our technical understanding of engineering implementation.

\section{CONCLUSION}

We have investigated several different methods for science camera calibration for extreme adaptive optics systems. The precision wave front sensor tasked with this activity will need to sense the residual wave front errors that would otherwise limit the performance of the system. We propose a fundamental shift in the architectures of existing adaptive optics systems by implementing a tight coupling between the Lyot coronagraph, precision wave front sensor, and the science camera. We explored options with the PWFS before and after the Lyot-coronagraph. We have selected a preliminary architecture that locates the PWFS post-coronagraph. This architecture minimizes the non-common path wave front errors, and takes advantage of the coronagraphs intrinsic sensitivity to wave front errors. A novel method for introducing diversity into the system gives us the ability to solve for the residual amplitude and phase errors in the residual wave front.

\section{ACKNOWLEDGEMENTS}

This work was carried out by the Jet Propulsion Laboratory, California Institute of Technology, under contract with the National Aeronautics and Space Administration. This work has been supported in part or full by the National Science Foundation Science and Technology Center for Adaptive Optics, managed by the University of California at Santa Cruz under cooperative agreement No. AST-9876783

\section{REFERENCES}

1. M. Troy, et.al., Palomar adaptive optics project: status and performance, Proc. SPIE, 4007 (2000), p. 31.

2. B. Macintosh, et.al., Extreme adaptive optics planet imager, XAOPI; Proc. SPIE 5169, 1, 2003.

3. Colucci, D., Atmospheric wavefront sensing and correction including the stellar phase shifting interferometer, Ph. D. Thesis, University of Arizona, 1993.

4. K. Creath, "Phase-measurement interferometry techniques," in Progress in Optics XXVI, E. Wolf, ed. (Elsevier, New York, 1988), Chap. 5.

5. J. Goodman, Introdution to Fourier Optics, $2^{\text {nd }}$ Ed., p.220, McGraw-Hill, 1996.

6. F. Zernike. Das Phasenkontrastverfahren bei der Mikroskopischen beobachtung. Z. Tech. Phys., 16:454, 1935.

7. S. Shaklan, D. Moody, and J. Green. Residual Wave Front Phase Estimation in the Reimaged Lyot Plane for the Eclipse Coronagraphic Telescope. Proc. SPIE vol. 4860 (Waikaloa, 2002).

8. Angel, J.R.P., Ground-based imaging of extrasolar planets using adaptive optics, Nature, 368, 203-207 (1994). 